\title{
Human cytomegalovirus UL141 protein interacts with CELF5 and affects viral DNA replication
}

\author{
FEI ZOU ${ }^{1,2^{*}}$, ZHI-TAO LU ${ }^{3 *}$, SHUANG WANG ${ }^{1}$, SI WU ${ }^{1}$, YING-YING WU ${ }^{1}$ and ZHENG-RONG SUN ${ }^{1}$ \\ ${ }^{1}$ Department of BioBank, Affiliated Shengjing Hospital of China Medical University, Shenyang, Liaoning 110004; \\ ${ }^{2}$ Department of Pediatrics, First Hospital of Jilin University, Changchun, Jilin 130021; ${ }^{3}$ Department of \\ Pediatrics, Zhangjiagang First People's Hospital, Zhangjiagang, Jiangsu 215600, P.R. China
}

Received June 14, 2017; Accepted January 5, 2018

DOI: $10.3892 / \mathrm{mmr} .2018 .8419$

\begin{abstract}
Human cytomegalovirus (HCMV) infection is the primary viral cause of congenital abnormalities and mental retardation in newborns. The HCMV UL141-encoded glycoprotein has been previously revealed to inhibit the cell-surface expression of cluster of differentiation (CD)155, CD122, tumor necrosis factor-related apoptosis-inducing ligand death (TRAIL)-receptor 1 (R1) and TRAIL-receptor 2 (R2), thus protecting virally-infected cells by allowing them to escape natural killer cell-mediated cytotoxicity. The present study investigated the interaction between HCMV UL141 and human fetal brain cDNA to elucidate the possible effects of UL141 on the nervous system. The findings of the current study demonstrate that the HCMV UL141 protein directly interacts with the human protein CUGBP Elav-like family member 5 (CELF5) via yeast two-hybrid screening, this interaction was confirmed by glutathione S-transferase pull-down and co-immunoprecipitation assays. Additionally, the present study demonstrated that the UL141 protein co-localizes with CELF5 in the cytoplasm of 293 cells using fluorescence confocal microscopy. CELF5 overexpression in a stably-expressing cell line significantly increased viral DNA copy number and titer in HCMV-infected U373MG cells. However, reducing CELF5 expression via specific small interfering RNAs did not affect viral DNA copy number or titer in HCMV-infected cells. The current findings suggest that the interaction between UL141 and CELF5 may be involved in modulating viral DNA synthesis and progeny production. Therefore, CELF5 may represent a possible mechanism for
\end{abstract}

Correspondence to: Mrs. Zheng-Rong Sun, Department of BioBank, Affiliated Shengjing Hospital of China Medical University, 36 Sanhao Street, Heping, Shenyang, Liaoning 110004, P.R. China

E-mail: sunzr@sj-hospital.org

${ }^{*}$ Contributed equally

Key words: human cytomegalovirus, UL141 protein, CELF5, human fetus brain, cDNA library regulation of HCMV genomic DNA synthesis, which is a key step during HCMV infection leading to neurological disease.

\section{Introduction}

Human cytomegalovirus (HCMV) is a ubiquitous herpes virus with infection rate of $\sim 50-80 \%$ in females of reproductive age from different regions of the world, including Australia, Canada, United States, Sweden, Finland, Spain, United Kingdom and Ghana (1). Following an HCMV infection, the primary clinical manifestations in healthy people are asymptomatic recessive or latent infections. When the immune system function is reduced, in the event of pregnancy, cancer, HIV infection, organ transplantation and childbirth, HCMV is activated from its latent infection status and proliferates to a primary or secondary infection. It may also lead to serious illnesses, including interstitial pneumonia, encephalitis, hepatitis, retinal failure and/or nervous systems, gastrointestinal diseases and stillbirth. Previous studies have reported that HCMV may also be associated with the occurrence of atherosclerosis and malignant tumors $(2,3)$.

Currently, the pathogenesis of HCMV infection remains to be elucidated. Previous studies revealed that the viral genetic differences, which are associated with the virulence, the tropism to tissues, and the ability to escape the immune system, may be the key factors determining whether HCMV infections lead to fetal and organ deformities (4,5). Primary clinical isolates carry at least 19 additional genes within the UL/b' genomic region (UL133-151 locus) that have been lost in several commonly-used HCMV strains, which have been passaged extensively in tissue culture (6). The UL141 gene within the UL/b' genomic region is highly conserved between HCMV isolates, encoding a protein containing a potential signal peptide, a hydrophobic transmembrane domain and three potential N-linked glycosylation sites. The UL141-encoded protein is a glycoprotein doublet with molecular masses of $37-40 \mathrm{kDa}$ that has been localized to the endoplasmic reticulum by immunofluorescence (7). HCMV UL141 is also requited to inhibit cell surface expression of cluster of differentiation (CD)155, a ligand for the activating receptor CD226 (7). UL141 requires assistance from additional HCMV-encoded functions to suppress CD112 expression, also termed nectin cell adhesion molecule 2 , by proteasome-mediated degradation (8). A previous study revealed that HCMV 
UL141 protein may bind tumor necrosis factor-related apoptosis-inducing ligand death (TRAIL)-receptor 1 (R1) and TRAIL-receptor 2 (R2) to inhibit apoptosis of infected cells (9). These studies revealed UL141 may effectively participate in HCMV immune escape through multiple mechanisms $(8,9)$. When CD155 and TRAIL-R2 accumulate in the endoplasmic reticulum, CD112 is effectively downregulated (8). A previous study indicated that HCMV UL141 requisitions US2 to target CD112 for proteasome degradation and modulates multiple immune-associated pathways (10). It has an important effect on cellular processes, such as antigen presentation, natural killer (NK) cell activity, cell migration and coagulation. In addition to acting as an immune evasion gene, UL141 is an inhibitor of HCMV proliferation in epithelial cell culture (7). A recent study suggested that all known mutations in the UL/b' genomic region in epithelial cells involve loss or mutation of UL141 (11). The interactions between UL141 and human proteins remain to be fully elucidated. Therefore, the present study performed a yeast two-hybrid screen to identify binary interactions that may yield important information regarding the process of viral entry and replication.

In the present study, the human protein CUGBP Elav-like family member 5 (CELF5) was identified as a directly-interacting partner of HCMV-pUL141. Additionally, the viral DNA level was increased in HCMV-infected U373 cells overexpressing CELF5. However, reduced expression of CELF5 had no significant effect on HCMV DNA synthesis. These findings suggest that CELF5 has a key role in modulating viral DNA synthesis. The interactions identified between HCMV UL141 and the CELF5 human protein may elucidate the molecular mechanisms underlying HCMV diseases.

\section{Materials and methods}

Oligonucleotides and plasmid constructs. Oligonucleotide primers used for the current study were purchased from Invitrogen; Thermo Fisher Scientific, Inc. (Waltham, MA, USA) and the recombinant plasmids used are presented in Table I. To generate pGBKT7-UL141 for the yeast two-hybrid screen, the coding sequence of HCMV UL141 was amplified by polymerase chain reaction (PCR) (12) using HCMV H strain DNA (Genbank no. GQ981646) as a template with the pGBKT7-UL141 primers (the restriction enzyme sites and protected bases are underlined): Forward (F) 5'-CCGGGC CATGGAGGCCGACATCGCCGAAAAGATGTGGG-3' and reverse (R) 5'-CGCGTCGACTCACCTCTTCATCTTTCT AACAC-3', obtained from Invitrogen (Thermo Fisher Scientific, Inc.), and then inserted into SfiI/SalI (Takara Biotechnology Co., Ltd., Dalian, China)-digested plasmid pGBKT7 (Clontech Laboratories, Inc., Mountain View, CA, USA). The inserted sequence of pGBKT7-UL141 was confirmed by sequencing (Shanghai Invitrogen Biotechnology Co., Ltd., Shanghai, China).

For the glutathione S transferase (GST) pull-down assay, the sequence of pACT2-CELF5, which was a candidate pUL141-interacting protein, was cloned into GST-tagged pGEX-4T-2 vector (Clontech Laboratories, Inc.) in EcoRI and XhoI (Takara Biotechnology Co., Ltd.) sites by electroporation (Bio-Rad Laboratories, Inc., Hercules, CA, USA) according to the manufacturer's protocol in BL21 (DE3) cells (Tiangen Biotech Co., Ltd., Beijing, China) at a density of $6 \times 10^{7}$ cells $/ \mathrm{ml}$, and expressed protein was termed GST-CELF5. GST-labeled CELF5 (GST-CELF5) was expressed in BL21 (DE3) cells (Tiangen Biotech Co., Ltd., Beijing, China) in logarithm growth period transfected with pGEX-4T-2-CELF5 by electroporation (Bio-Rad Laboratories, Inc.) according to the protocol provided by the manufacturer and induced with isopropyl $\beta$-D-1-thiogalactopyranoside (IPTG; Clontech Laboratories, Inc., Mountainview, CA, USA). For the co-immunoprecipitation (co-IP) assays, the sequences of UL141 and CELF5 were inserted into Myc- and HA-tagged pCMV vector (Clontech Laboratories, Inc.) for expression in 293T cells.

To generate pEGFP-UL141 and pDsRed-CELF5 constructs, which were used to express fluorescently-tagged fusion proteins in human cells, the sequences of UL141 and CELF5 were amplified using the primers UL141 F, 5'-CG $\underline{\text { GAATTCC }}$ TGCCGCCGGGAGTCGCTCCG-3' and R, 5'-CGCGGATCC TCACCTCTTCATCTTTCTAA-3'; CELF5 F, 5'-CGGAAT TCCCCTCACATTGCCCTTCAG-3' and R 5'-CGCGGATCC ACCTTCTCGCTGCTGCTG-3', then inserted into pEGFP and pDsRed vectors (Clontech Laboratories, Inc.), respectively. The resultant constructs were confirmed by restriction digestion profiles and sequencing (Shanghai Invitrogen Biotechnology Co., Ltd., Shanghai, China).

Viruses, antibodies and cells. In this study, 10 patients (6 male, 4 female) aged $<5$ months in the Affiliated Shengjing Hospital of China Medical University who met the CMV infection diagnostic criteria (13) were enrolled between December 2006 and March 2007. The urine of the patient was collected and treated with an antibiotic cocktail containing $500 \mathrm{U} / \mathrm{ml}$ penicillin, $500 \mu \mathrm{g} / \mathrm{ml}$ streptomycin and $500 \mu \mathrm{g} / \mathrm{ml} \mathrm{kanamycin}$. Following centrifugation at $300 \mathrm{x} g$ for $15 \mathrm{~min}$, the supernatant was applied onto a human embryonic lung fibroblasts (HELFs) monolayer, and incubated at $37^{\circ} \mathrm{C}$ overnight to allow virus adsorption. Then the inoculum was removed and the cells washed with Hank's buffer. The inoculated HELFs were maintained at $37^{\circ} \mathrm{C}$ in Modified Eagle's medium (Gibco; Thermo Fisher Scientific, Inc.) supplemented with $2 \%$ fetal bovine serum $(\mathrm{FBS})$, penicillin $(100 \mathrm{U} / \mathrm{ml}) /$ streptomycin $(100 \mu \mathrm{g} / \mathrm{ml})$ and $2 \mathrm{mM}$ l-glutamine (Gibco; Thermo Fisher Scientific, Inc.). The culture medium was refreshed every 5 days and the cells passaged when necessary. The cultures were observed every other day to monitor appearance of cytopathic effects (CPEs). When HCMV characteristic CPEs appeared, the culture was cultured for an additional 3 days and then passaged. A low-passage clinical isolate Chinese HCMV strain Han was thus successfully isolated from these urine samples. All participating patients gave their written informed consent prior to enrollment in the study. This study was approved by the Ethics Committee of the Affiliated Shengjing Hospital of China Medical University (Shenyang, China) and conducted in accordance with the principles of the Declaration of Helsinki. The virus was propagated in MRC-5 cells which were obtained from the Type Culture Collection of the Chinese Academy of Sciences (Shanghai, China). Monoclonal mouse anti-human antibodies against c-Myc (1:1,000; cat. no. AHO0052; Thermo Fisher Scientific, Inc.) or polyclonal goat anti-human antibodies against GST (1:1,000; cat. no. PA5-18394; Thermo Fisher Scientific, Inc.) were used for western blot analysis. Goat anti-mouse horseradish peroxidase 
Table I. Plasmid constructs used in the current study.

\begin{tabular}{|c|c|c|}
\hline Plasmid & Description & Reference/source \\
\hline pGEX-4T-2 & $\begin{array}{l}\text { Cloning vector for protein expression fused with GST tag in } \\
\text { Escherichia coli cells }\end{array}$ & Clontech Laboratories, Inc. \\
\hline pGEX- CELF5 & pGEX-4T-2 containing full-length human CELF5-encoding sequence & This study \\
\hline pGBKT7 & $\begin{array}{l}\text { Cloning vector for protein expression fused with the GAL4 DNA-binding } \\
\text { domain in yeast }\end{array}$ & Clontech Laboratories, Inc. \\
\hline pGBKT7-UL141 & pGBKT7 containing HCMV UL141 full length sequence & This study \\
\hline pACT2 & $\begin{array}{l}\text { Cloning vector for protein expression fused with GAL4 activation domain } \\
\text { in yeast }\end{array}$ & Clontech Laboratories, Inc. \\
\hline pACT2-CELF5 & pACT2 containing full-length human CELF5-encoding sequence & This study \\
\hline pEGFP & $\begin{array}{l}\text { Cloning vector for protein expression with green fluorescent protein(GFP) } \\
\text { in mammalian cell }\end{array}$ & Clontech Laboratories, Inc. \\
\hline pEGFP-UL141 & pEGFP containing HCMV UL141 full length sequence & This study \\
\hline pDsRed & $\begin{array}{l}\text { Cloning vector for protein expression with red fluorescent protein(RFP) in } \\
\text { mammalian cell }\end{array}$ & Clontech Laboratories, Inc. \\
\hline pDsRed-CELF5 & pDsRed containing full-length human CELF5-encoding sequence & This study \\
\hline pCMV-Myc & $\begin{array}{l}\text { Cloning vector for protein expression fused with c-Myc tag in } \\
\text { mammalian cell }\end{array}$ & Clontech Laboratories, Inc. \\
\hline pCMV-Myc-UL141 & pCMV-Myc containing HCMV UL141 full length sequence & This study \\
\hline pCMV-HA & $\begin{array}{l}\text { Cloning vector for protein expression fused with hemagglutinin (HA) tag } \\
\text { in mammalian cell }\end{array}$ & Clontech Laboratories, Inc. \\
\hline pCMV-HA-CELF5 & pCMV-HA containing full-length human CELF5-encoding sequence & This study \\
\hline pACT2-cDNA & pACT2 containing a human fetal brain cDNA library & This study \\
\hline
\end{tabular}

(HRP)-conjugated (1:2,000; cat. no. A0216; Beyotime Institute of Biotechnology, Haimen, China) and rabbit anti-goat HRP-conjugated immunoglobulin G secondary antibodies (1:2,000; cat. no. abs20005; Absin Bioscience, Inc., Shanghai, China) were used for detection. Astrocytoma U373MG cells (provided by Professor Songya Lv, State Key Laboratory of Virology, College of Life Sciences, Wuhan University, Wuhan, China, source American Type Culture Collection, Manassas, VA, USA), human embryonic lung fibroblast MRC-5 cells, and 293T cells (Shanghai Institute of Biochemistry and Cell Biology) were cultured in Dulbecco's modified Eagle's medium (DMEM; GE Healthcare Life Sciences, Logan, UT, USA) containing $10 \%$ fetal calf serum (GE Healthcare Life Sciences), $1 \%$ penicillin and $1 \%$ streptomycin. The U373MG cells may be contaminated/mis-identified and are likely derived from U-251MG cells. To generate a U373MG cell line that transiently expressed CELF5 (U373-S) and control cells, pDsRed-C1-CELF5 and an empty vector pDsRed-C1 were transfected into U373 cells using Lipofectamine ${ }^{\circledR}$ LTX with Plus $^{\mathrm{TM}}$ Reagent, according to the manufacturer's protocol (Invitrogen; Thermo Fisher Scientific, Inc.). The two cell lines were seeded at a density of $1 \times 10^{6}$ in 6 -well plates prior transfection. The levels of CELF5 in individual cell clones were determined by western blot analysis.

Yeast two-hybrid screening. Yeast two-hybrid experiments were performed according to the manufacturer's protocol using the Matchmaker GAL4 Two-Hybrid System 3 (Clontech Laboratories, Inc.). Plasmid pGBKT7-UL141 containing the GAL4 DNA-binding domain was transformed into
Saccharomyces cerevisiae AH109 (Clontech Laboratories, Inc.,) containing a human fetal brain cDNA library, pACT2-cDNA. The human fetal brain cDNA library was provided by Professor Gengfu Xiao from the State Key Laboratory of Virology, College of Life Sciences, Wuhan University (Wuhan, China), which was cloned into pACT2 (Clontech Laboratories, Inc.) by electroporation (Bio-Rad Laboratories, Inc.) according to the manufacturer's protocol in BL21 (DE3) cells (Tiangen Biotech Co., Ltd., Beijing, China) at a density of $6 \times 10^{7}$ cells $/ \mathrm{ml}$ in logarithmic growth period. Positive colonies were selected on synthetic dropout medium (Clontech Laboratories, Inc.,) lacking tryptophan, leucine, adenine and histidine and confirmed by detecting $\alpha$-galactosidase (Clontech Laboratories, Inc.) activity as previously described (14). The cells that produced blue signals on a colony lift assay were identified and defined as positive colonies. Subsequently, plasmids containing the coding sequence for UL141-interacting partners (termed pACT2-cDNA) were extracted from positive colonies and co-transformed into AH109 with the pGBKT7-UL141 in order to validate the interaction. Human gene sequences in pACT2-cDNA were determined from these selected colonies with the pACT2 vector sequencing primer (5'-AATACC ACTACAATGGAT-3') and matched with the Blast network service at the National Center for Biotechnology Information (http://www.ncbi.nlm.nih.gov/blast).

GST pull-down and western blot analysis. GST pull-down experiments were performed using the MagneGST ${ }^{\mathrm{TM}}$ Pull-down system following the manufacturer's protocol 
(Promega Corporation, Madison, WI, USA). c-Myc-labeled UL141 protein was synthesized as a prey protein in vitro using pGBKT7-UL141 in a TNT (quick coupled transcription/translation reaction) $\mathrm{T} 7$ Quick reaction (Promega Corporation) according to the manufacturer's protocol (15). GST fusion protein (GST-CELE5) expression was induced in $E$. coli BL21 (DE3) with $5 \mathrm{mmol} /$ lisopropyl $\beta$-D-thiogalactoside (Clontech Laboratories, Inc.). After allowing $4 \mathrm{~h}$ of post-induction, cell lysates were harvested to collect the bait protein GST-CELF5. GST-CELF5 was immobilized onto MagneGST particles and incubated with the c-Myc-labeled UL141 protein at $25^{\circ} \mathrm{C}$ for $1.5 \mathrm{~h}$ on a rotating platform. After washing three times with a buffer, proteins binding the MagneGST particles were eluted and solubilized in SDS sample buffer (Beyotime Institute of Biotechnology).

To perform western blot analysis, denatured proteins were subjected to electrophoresis on a $12 \%$ SDS-polyacrylamide (Sigma-Aldrich; Merck KGaA, Darmstadt, Germany) and transferred electronically onto polyvinylidene fluoride membranes (Sigma-Aldrich, Merck Millipore, Darmstadt, Germany). Membranes were blocked in 5\% non-fat dried milk in tris-buffered saline (TBS) and 0.05\% Tween-20. Detection of tagged proteins was performed following incubation overnight at $4^{\circ} \mathrm{C}$ with 1:1,000-diluted mouse anti-Myc or 1:500-diluted mouse anti-GST primary antibodies followed by incubation for $2 \mathrm{~h}$ at room temperature with 1:2,000-diluted goat anti-mouse IgG secondary antibody conjugated with HRP (Beyotime Institute of Biotechnology). Proteins were subsequently visualized using enhanced chemiluminescence detection reagent kits (Thermo Fisher Scientific, Inc.) and signals were detected with the Molecular Imager ChemiDoc XRS system (Bio-Rad Laboratories, Inc.).

Co-IP and western blot analysis. 293T cells at a density of $2 \times 10^{6}$ cells/plate were cultured in DMEM containing $10 \%$ fetal calf serum in $60 \mathrm{~mm}$ plates prior transfection. At $\sim 90 \%$ confluence, cells were co-transfected with pCMV-Myc-UL141 and pCMV-HA-CELF5 with using Lipofectamine ${ }^{\circledR} 2000$ (Invitrogen; Thermo Fisher Scientific, Inc.). At $48 \mathrm{~h}$ post-transfection, cell lysates were harvested and proteins were extracted with M-PER ${ }^{\mathrm{TM}}$ Mammalian Protein Extraction Reagent (Thermo Fisher Scientific, Inc.). Co-immunoprecipitation experiments were performed using the ProFound mammalian c-Myc tag immunoprecipitation/co-immunoprecipitation (IP/co-IP) and HA tag IP/co-IP kits, following the manufacturer's protocols (cat. nos. 23620 and 23610 respectively; Pierce; Thermo Fisher Scientific, Inc.). Myc-UL141 and HA-CELF5 were detected by western blotting as aforementioned using mouse anti-Myc or rabbit anti-HA antibodies (cat. no. 71-5500; 1:100; Thermo Fisher Scientific, Inc.) incubated overnight at $4^{\circ} \mathrm{C}$ and corresponding peroxidase-conjugated goat anti-mouse and goat anti-rabbit (cat. no. A0208; 1:2,000; Beyotime Institute of Biotechnology) IgG secondary antibodies incubated at room temperature for $2 \mathrm{~h}$. The immunoreactive bands were visualized by the diaminobenzidine coloration method. The semi-quantitation of proteins was surveyed with a Tanon GIS gel imager System.

Immunofluorescent co-localization. $293 \mathrm{~T}$ cells $\left(2 \times 10^{6}\right)$ were cultured on glass coverslips in DMEM containing $10 \%$ fetal calf serum. They were seeded into a $35-\mathrm{mm}$ confocal microscope dish (Nest Biotechnology Co., Ltd., Jiangsu, China) 24 h prior to transfection. At $\sim 75 \%$ confluence, cells were co-transfected with $4 \mu \mathrm{g}$ pEGFP-UL141 and $4 \mu \mathrm{g}$ pDsRed-CELF5 using Lipofectamine $^{\circledR} 2000$ (Invitrogen; Thermo Fisher Scientific, Inc.). At $48 \mathrm{~h}$ post-transfection, cells were subjected to DAPI staining (Invitrogen; Thermo Fisher Scientific, Inc.) for $5 \mathrm{~min}$ at room temperature, and the expression levels of EGFP-UL141 and DsRed-CELF5 were detected using a TCS SP2 Leica laser scanning confocal microscope (Nikon Eclipse C1 Plus; Nikon Corporation, Tokyo, Japan) with a $488 \mathrm{~nm}$ and a $543 \mathrm{~nm}$ excitation beam.

Transfection small interfering (si)RNA into cells. U373-S cells ( $2 \times 10^{5}$ cells/well) were seeded in 12 -well plates and transfected with anti-CELF5 siRNA (CELF5-siRNA; Ambion; Thermo Fisher Scientific, Inc.) and control siRNA (C-siRNA; Santa Cruz Biotechnology, Inc., Dallas, TX, USA). For each well, $3 \mu \mathrm{l}$ of $20 \mathrm{nM}$ siRNA and $3 \mu \mathrm{l}$ Lipofectamine RNAi MAX (Invitrogen; Thermo Fisher Scientific, Inc.) were diluted in 50 and $12 \mu \mathrm{l}$ Opti-MEM medium (Invitrogen; Thermo Fisher Scientific, Inc.), respectively. Following a 5 min incubation at room temperature, both solutions were mixed. After $20 \mathrm{~min}$, $400 \mu$ l pre-warmed Opti-MEM medium (Invitrogen; Thermo Fisher Scientific, Inc.) was added to each transfection reaction mixture, which was subsequently added to cells. At $10 \mathrm{~h}$ post-transfection, siRNA-containing medium was removed, and cells were washed and incubated with 10\% DMEM. The transfection procedure was subsequently repeated. Cells were either prepared for analysis by western blotting or HCMV infection $48 \mathrm{~h}$ after the second transfection.

Viral infection and HCMV DNA level analysis. To assay viral growth, the cells $\left(1 \times 10^{5}\right)$ were infected with HCMV at a multiplicity of infection $=3$, subsequently the cells and medium were collected at 5 days post-infection and viral stocks were prepared. The titers of the viral stocks were determined by infecting $1 \times 10^{5}$ human foreskin fibroblasts and the number of plaques 10-14 days following the infection were counted. The values obtained were averages from triplicate experiments. The levels of intracellular viral DNA were quantified by amplifying the HCMV UL83 sequence with its primers (forward, 5'-GTCAGCGTTCGTGTTTCCCA-3' and reverse, 5'-GGGACACAACACCGTAAAGC-3') using quantitative PCR (qPCR). The number of viral genomes was normalized to the number of cellular copies of $\beta$-actin as determined using $\beta$-actin primers (forward, 5'-CGGAACCGCTCATT GCC-3' and reverse, 5'-ACCCACACTGTGCCCATCTA-3'). Amplification was performed in a $25 \mu 1$ reaction mixture, which contained $4 \mu \mathrm{l}$ DNA extract, $12.5 \mu$ l Power SYBR-Green PCR master mix (Applied Biosystems; Thermo Fisher Scientific, Inc.) and $0.5 \mu \mathrm{l}$ each primer at $10 \mu \mathrm{M}$ using an ABI 7300 device (Applied Biosystems; Thermo Fisher Scientific, Inc.). Thermal cycling conditions were as follows: $95^{\circ} \mathrm{C}$ for $10 \mathrm{~min}, 40$ cycles of $95^{\circ} \mathrm{C}$ for $15 \mathrm{sec}$ and $60^{\circ} \mathrm{C}$ for $1 \mathrm{~min}, 95^{\circ} \mathrm{C}$ for $15 \mathrm{sec}, 60^{\circ} \mathrm{C}$ for $1 \mathrm{~min}$ and $95^{\circ} \mathrm{C}$ for $15 \mathrm{sec}$. qPCR results were derived from three independent experiments using the $2^{-\Delta \Delta \mathrm{Cq}}$ method (16).

Statistical analysis. Statistical significance between different groups was determined by one-way analysis of variance test with post hoc Bonferroni correction. All data were collected 
and analyzed with SPSS version 13.0 (SPSS, Inc., Chicago, IL, USA). GraphPad Prism version 5 (GraphPad Software, Inc., La Jolla, CA, USA) was used to produce the correlation graphs. $\mathrm{P}<0.05$ was considered to indicate a statistically significant difference.

\section{Results}

Interaction between UL141 and CELF5 identified by yeast two-hybrid analysis. Yeast two-hybrid analysis was used to identify potential protein partners that interact with $\mathrm{HCMV}$ UL141. The candidate, PACT2-CELF5, was screened to examine its interaction with UL141 and the nucleotide sequence of pACT2-CELF5 was $100 \%$ identical to that of the human CELF5 sequence in NCBI.

Interaction between HCMV pUL141 and CELF5 identified by GST pull-down assay. GST pull-down experiments were performed in order to validate whether there is a direct interaction between HCMV pUL141 and CELF5 in vitro. In these experiments, GST-tagged CELF5 was used as a bait protein and c-Myc-tagged pUL141 as the prey protein. UL141 and CELF5 were detected as proteins of $\sim 37$ and $50 \mathrm{kDa}$, respectively by western blotting (Fig. 1). According to these findings, the UL141 protein has the ability to interact with the CELF5 protein in the absence of another medium in vitro.

Identification of the interaction between HCMV pUL141 and CELF5 in human cells by co-IP. In order to confirm the identified interaction between pUL141 and CELF5 in human cells, a co-IP assay was performed. In these experiments, the pCMV-Myc-UL141 and pCMV-HA-CELF5 plasmids, which expressed fusion proteins with an amino-terminal Myc or HA epitope tag, respectively were constructed by cloning the coding sequences for UL141 and CELF5 into the expression vectors pCMV-Myc and pCMV-HA, respectively. 293 cells were transfected with the expression constructs and were harvested after $48 \mathrm{~h}$. Protein lysates from the cells were initially immunoprecipitated with anti-Myc or anti-HA and subsequently immunoblotted with antibodies against the Myc and HA epitope tags. HCMV UL141 and CELF5 were detected as proteins of 37 and $25 \mathrm{kDa}$ with anti-c-Myc and anti-HA antibodies, respectively, by western blotting (Fig. 2). These findings confirm the interaction between viral UL141 and host CELF5 proteins in human cells.

Identification of pUL141 and CELF5 co-localization in human cells by fluorescence confocal microscopy. If UL141 is associated with CELF5 in cells, it was expected that these proteins would localize within the same cellular compartments. To determine whether UL141 co-localizes with CELF5, 293 cells were transfected with pEGFP-UL141 and/or pDsRed-CELF5 plasmids. The cellular localization of those expressed proteins was investigated using fluorescence confocal microscopy. In cells transfected with construct GFP-UL141 alone, UL141 was found to be primarily expressed in the cytoplasm (Fig. 3A), whereas RPF-CELF5 was primarily localized in the cytoplasm and nucleus of 293 cells (Fig. 3B). Co-localization in the cytoplasm of 293 cells was observed for these proteins in a diffuse pattern and within the nucleus in a mottled pattern

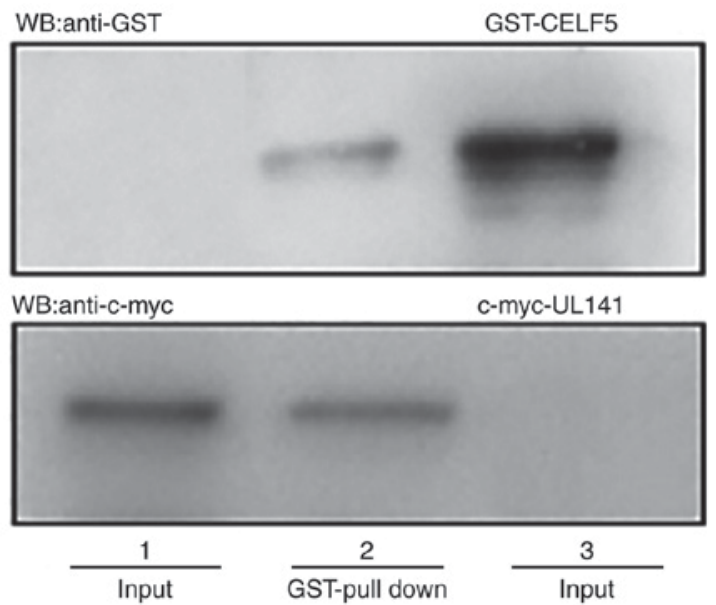

Figure 1. Identification of the interaction between CELF5 and HCMV pUL141 protein by GST pull-down analysis. The c-Myc-labeled UL141 protein was synthesized as a prey protein in vitro by using the pGBKT7-UL141 plasmid in a TNT T7 Quick reaction and was assayed for WB using anti-c-Myc antibodies (lane 1). The mix of c-Myc-tagged pUL141 and GST-CELF5 (lane 2) were captured by the MagneGST particles and detected by anti-c-Myc antibodies and anti-GST antibodies, respectively. The GST-fusion protein (GST-CELE5), which was induced to express in E.coli BL21 as a bait protein was detected by anti-GST antibodies (lane 3). GST, glutathione S-transferase; WB, western blotting; CELF5, CUGBP Elav-like family member 5; HCMV, human cytomegalovirus; c-Myc, c-Myc proto-oncogene.
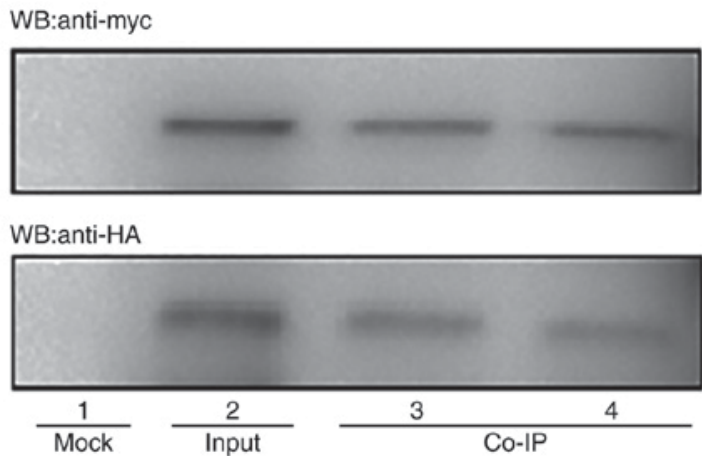

Figure 2. Interaction between c-Myc-tagged pUL141 and HA-tagged CELF5 was identified by co-IP. 293 cells were co-transfected with plasmids pCMV-Myc-UL141 and pCMV-HA-CELF5. Cell lysates were harvested $48 \mathrm{~h}$ after transfection. The negative control (lane 1), input protein samples (lane 2) and samples that were either immunoprecipitated with c-Myc (lane 3) or HA antibodies (lane 4) were separated on SDS-PAGE gel and analyzed with western blotting using anti-Myc and anti-HA antibodies, respectively. The Myc-tagged UL141 and HA-tagged CELF5 protein were detected in the captured analysis. IP, immunoprecipitation; HA, hemagglutinin; c-Myc, c-Myc proto-oncogene; CELF5, CUGBP Elav-like family member 5.

(Fig. 3C). At $48 \mathrm{~h}$ post transfection, nuclear DNA was stained with DAPI (blue image; Fig. 3D).

Effect of up and downregulation of CELF5 expression on HCMV DNA synthesis and lytic infection. In order to investigate the effect of CELF5 on HCMV DNA replication, U373MG cells were transfected with pCMV-HA-CELF5 to induce overexpression of exogenous CELF5 (U373-Celf5) or transfected with siRNA-CELF5 to downregulate the expression of endogenous CELF5 (Celf5-siRNA). Different cells, including U373, U373-Celf5, Celf5-siRNA, and C-siRNA groups were infected with HCMV and then harvested $48 \mathrm{~h}$ post-infection. 

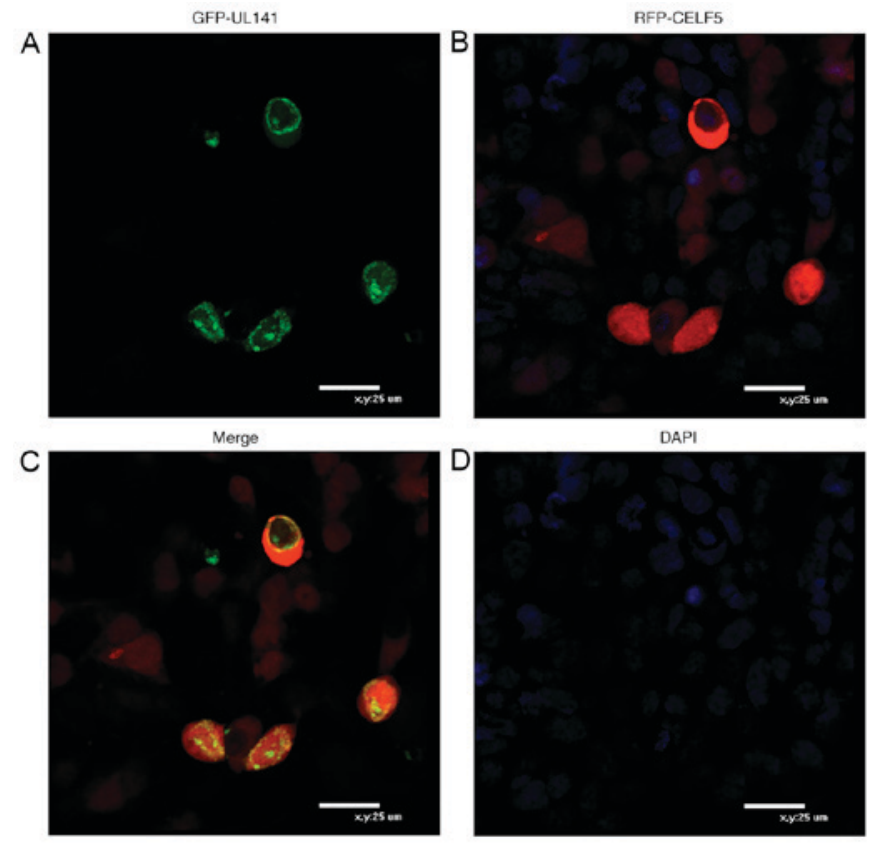

Figure 3. Co-localization of UL141 and CELF5 expressed in 293 cells 293 Cells were transfected with (A) pGFP-UL141, (B) pRPF-CELF5 or (C) both and fixed $48 \mathrm{~h}$ post-transfection. (D) DAPI (blue) was used for staining the nuclear DNA. Localization of the expressed proteins was observed using fluorescent confocal microscope. Scale bar, $25 \mu \mathrm{m}$. CELF5, CUGBP Elav-like family member 5; GFP, green fluorescent protein; RPF, red fluorescent protein.

CELF5 expression levels were confirmed by western blot analysis. The expression of exogenous CELF5 in U373-Celf5 cells was higher compared with other cells (Fig. 4A). The expression level of the endogenous CELF5 protein in Celf5-siRNA cells collected $48 \mathrm{~h}$ post-HCMV infection was reduced by $>60 \%$ (data not shown) when compared with the cells transfected with control siRNA (C-siRNA; Fig. 4A).

To determine the effect of altered CELF5 expression on HCMV infection, total intracellular DNA from different cell types was extracted $48 \mathrm{~h}$ post-infection and the level of viral DNA synthesis was analyzed. The number of viral genomes present in each sample was detected by qPCR. Cellular $\beta$-actin was used as an internal reference to normalize the copy number of the viral UL83 sequence. Viral DNA copy numbers (UL83/ $\beta$-actin) were significantly increased by $\sim 3$-fold in the U373-Celf5 cells when compared with $\mathrm{C}$-siRNA $(\mathrm{P}<0.001)$ and parental U373MG cells $(\mathrm{P}<0.001)$ at $48 \mathrm{~h}$ post-infection (Fig. 4B). These findings suggest that upregulating CELF5 expression in HCMV-infected cells may promote HCMV DNA synthesis. However, no significant difference was identified in the number of viral DNA copies (UL83/ß-actin) among cells treated with anti-CELF5-siRNA (Celf5-siRNA) and control-siRNA or parental U373MG cells. This may be due to the low expression level of CELF5 in U373MG cells during viral infection. The interaction between HCMV pUL141 and CELF5 is limited in infected cells; therefore, interference in CELF5 expression levels may not have a significant effect on HCMV replication in U373MG cells.

\section{Discussion}

Previous studies on HCMV infection in newborns and infants, the patient's clinical symptoms, diagnosis, treatment and
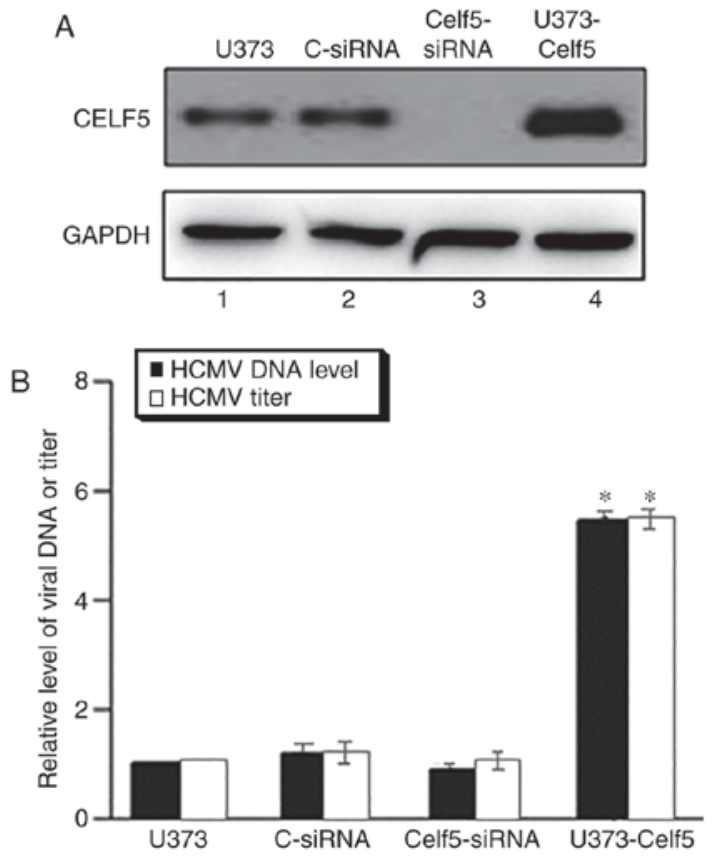

Figure 4. CELF5 levels affect HCMV DNA synthesis by quantitative polymerase chain reaction. U373MG cells were transfected with C-siRNA, CELF5-siRNA, or U373-CELF5. (A) Western blot analysis of the levels of CELF5 in the parental U373 cells, U373 cells that were transfected with C-siRNA or Celf5-siRNA, cells overexpressing exogenous CELF5. The expression of GAPDH was used as the internal reference. At $48 \mathrm{~h}$ post-transfection, cells were infected with HCMV, multiplicity of infection=3. CELF5 protein expression level was detected by western blot analysis with an anti-CELF5 antibody. (B) Total infection cultures were collected 4 days post-infection to assay the level of viral production. The values of the relative HCMV DNA levels and HCMV titer represent the ratios of the levels of viral DNA in different cells, to that in the parental U373MG cells. Data are presented as the mean \pm standard deviation of two independent experiments performed in triplicate. ${ }^{*} \mathrm{P}<0.001$ vs. Celf5-siRNA and vs. C-siRNA. CELF5-siRNA and control U373 cells. C, control; siRNA, small interfering RNA; U373-CELF5, pCMV-HA-CELF5; HCMV, human cytomegalovirus. CELF5, CUGBP Elav-like family member 5.

the influence of HCMV infection on immune function have attracted widespread attention (17-19). As with other herpes viruses, HCMV may establish a lifelong relationship with the host through active and latent infection. Previous studies demonstrated that in order to be able to survive and maintain a latent infection status for a long time, the virus encodes a variety of immune escape-associated proteins to target different antigen-presenting molecules, such as major histocompatibility complex (MHC)-I, MHC-II and inhibitory and activating receptors on NK cells following HCMV infection $(20,21)$. For example, HCMV proteins, such as US2, US3, US6, US10, US11, UL82 and UL83 may all suppress the expression of MHC-I and MHC-II on the cell surface through different mechanisms and thus regulate antigen presentation (20). UL141 promotes HCMV survival by blocking CD155, CD122, TRAIL-R1 and TRAIL-R2 surface expression, subsequently inhibiting apoptosis and NK cell-mediated host cell cytotoxicity $(22,23)$. These actions may be mediated by interactions between viral and human proteins. Understanding these interactions is crucial for the identification of the mechanisms of viral latent and proliferative infections.

In the present study, a yeast two-hybrid screen was used to identify novel human proteins that may interact with the HCMV UL141 protein. Preliminary findings indicated 6 candidates 
(Table II), one of which was CELF5. CELF5 is a member of the CELF or Bruno-like family and is involved in the control of mRNA splicing and translation. Human CELF5 expression is primarily located in the fore-, mid- and hindbrain, but is low in the corpus callosum and pons (24). However, its specific function remains to be determined. GST pull-down analysis indicated a physical interaction between CELF5 and HCMV pUL141 proteins (Fig. 1). A co-IP assay was used to validate this interaction in a cellular milieu. Myc-tagged UL141 proteins specifically immunoprecipitated with HA-tagged CELF5 (Fig. 2). Co-localization of CELF5 and UL141 protein was confirmed by confocal microscopy analysis. These proteins were primarily co-localized in the cytoplasm of 293 cells (Fig. 3). To the best of our knowledge, the present study is the first to report of a physical interaction between CELF5 and any HCMV protein.

The current study also demonstrated that an increase in CELF5 expression in HCMV-infected cells promoted HCMV DNA synthesis. The interaction between UL141 and CELF5 may be involved in modulating viral DNA synthesis and progeny production. The role of UL141 in gene expression has been previously suggested to be that UL141 promotes HCMV survival by inhibiting apoptosis and NK cell-mediated host cell cytotoxicity (7). It may be the reason for the findings observed in the current study. Conversely, reducing CELF5 mRNA expression via specific siRNA did not affect viral DNA copy number or titer in HCMV-infected cells (Fig. 4). This phenomenon may be due to the low basal expression level of CELF5 in U373MG cells during viral infection.

The CELF protein family contains 6 members termed CELF1 to CELF6. CELF proteins have 3 conserved RNA recognition motifs (RNP-1)-RRM1, RRM2 in the N-terminus of the protein, and RRM3 in the C-terminus. RRM3 is separated from RRM1 and RRM2 by a non-conserved or divergent domain that is unique in each family member (25). The CELF family of RNA binding proteins regulate several steps of RNA processing in the nucleus and cytoplasm, including pre-mRNA alternative splicing, $C$ to U RNA editing, deacetylation, mRNA decay and translation. CELF proteins have been have been previously revealed to have roles in gametogenesis and early embryonic development, heart and skeletal muscle function, and neurosynaptic transmission in vivo (26). In a study of pathogenesis of nervous system diseases, CLEF RNA processing was demonstrated to have a role in a variety of nervous system diseases, such as ankylosing muscular dystrophy, amyotrophic lateral sclerosis, ataxia and multiple neurofibromatosis type 1 (NF-1). CELF proteins are involved regulating a neurofibromin exon that may be important for regulating higher cognitive functions (27). Previous studies revealed that overexpression and siRNA knockdown of CELF1-5 in cell transfection experiments lead to a decrease or increase of NF1 exon 23a inclusion, respectively $(28,29)$. CELF1 has an important role in promoting the responses of immune cells to external stimulation and coordinating gene networks involved in cell activation (30). CELF5 has been previously described as a refined differentially expressed gene in a cross-species comparison including neurologically normal humans, rats and mice, and its transcript it was enriched in axons and synapses (31). It is possible that different CELF5 levels may impact upon the anatomy and physiology of motor neurons. However, CELF5-associated research is limited at
Table II.Homologous genes that interacted with HCMV UL141 protein were analyzed by comparing the gene sequences with the human genome from Genebank.

Homologous genes Homology $(\%)$

Homo sapiens chromodomain helicase 98

DNA binding protein 3 (CHD3)

Homo sapiens DnaJ (Hsp40) homolog 98 (DNAJB1)

Homo sapiens dachshund homolog 1 (DACH1)

Homo sapiens transducin-like enhancer of split1 (TLE1)

Homo sapiens wilms tumor 1 interacting protein (WTIP)

Homo sapiens CUGBP, Elav-like family member 5 (CELF5)

the mRNA level, and investigation of the host protein CELF5 also uncommon.

CELF proteins exhibit differential localizations that may be associated with specific neuronal subtype, developmental stage or pathogenic condition. CELF1 has been primarily identified in the nuclei of cultured neurons (32), whereas CELF5 was located predominantly in the cytoplasm (33). This is in line with the findings of the current study, that CELF5 and UL141 proteins are primarily co-localized in the cytoplasm of 293 cells. Although few functional models of CELF5 have been reported, CELF5 has been hypothesized to have an important role in neural development and function from its embryonic and postnatal expression patterns (25). It may also be associated with central nervous system damage $(25,28)$. However, a specific functional mechanism remains to be elucidated.

CELF proteins are also associated with apoptosis. The present study determined that CELF5 may promote HCMV replication, which is a key step during persistent infections. It has been previously hypothesized that CELF5 as a member of CELF family proteins may also inhibit apoptotic factors. For example, CELF1 overexpression may inhibit apoptosis in tumor cells and its downregulation may lead to sensitivity in tumor cells, resulting in death receptor-mediated apoptosis (31). Therefore, the current study suggests that future should aim to identify the impact of CELF5 expression in specific cellular pathways associated with nervous system damage caused by HCMV. However, reducing the expression of endogenous CELF5 had no significant effect on HCMV replication in U373MG cells, which may be associated with low endogenous expression of CELF5 in U373 cells. Therefore, its specific mechanism of action requires further investigation. The current findings suggest that the interaction between UL141 and CELF5 may be involved in the modulation of viral DNA synthesis and progeny production. Therefore, CELF5 may represent a possible mechanism for regulating HCMV genomic DNA synthesis, which is a key step during HCMV infection leading to neurological disease. Future studies are required to elucidate the mechanism of the interaction between pUL141 and CELF5 in the regulation of HCMV DNA replication. 


\section{Acknowledgements}

The current study was supported by the National Natural Science Foundation of China (grant no. 81171581) and the Outstanding Scientific Fund of Shengjing Hospital.

\section{References}

1. Manicklal S, Emery VC, Lazzarotto T, Boppana SB and Gupta RK: The 'silent' global burden of congenital cytomegalovirus. Clin Microbiol Rev 26: 86-102, 2013.

2. Huang ZR, Yu LP, Yang XC, Zhang F, Chen YR, Feng F, Qian XS and Cai J: Human cytomegalovirus linked to stroke in a Chinese population. CNS Neurosci Ther 18: 457-460, 2012.

3. Dziurzynski K, Chang SM, Heimberger AB, Kalejta RF, McGregor Dallas SR, Smit M, Soroceanu L and Cobbs CS; HCMV and Gliomas Symposium: Consensus on the role of human cytomegalovirus in glioblastoma. Neuro Oncol 14: 246-255, 2012.

4. Sinzger C, Digel M and Jahn G: Cytomegalovirus cell tropism. Curr Top Microbiol Immunol 325: 63-83, 2008.

5. Gredmark-Russ S and Söderberg-Nauclér C: Dendritic cell biology in human cytomegalovirus infection and the clinical consequences for host immunity and pathology. Virulence 3: 621-634, 2012

6. Dolan A, Cunningham C, Hector RD, Hassan-Walker AF, Lee L, Addison C, Dargan DJ, McGeoch DJ, Gatherer D, Emery VC, et al: Genetic content of wild-type human cytomegalovirus. J Gen Virol 85: 1301-1312, 2004.

7. Tomasec P, Wang EC, Davison AJ, Vojtesek B, Armstrong M, Griffin C, McSharry BP, Morris RJ, Llewellyn-Lacey S, Rickards C, et al: Downregulation of natural killer cell-activating ligand CD155 by human cytomegalovirus UL141. Nat Immunol 6: $181-188,2005$

8. Prod'homme V, Sugrue DM, Stanton RJ, Nomoto A, Davies J, Rickards CR, Cochrane D, Moore M, Wilkinson GW and Tomasec P: Human cytomegalovirus UL141 promotes efficient downregulation of the natural killer cell activating ligand CD112. J Gen Virol 91: 2034-2039, 2010.

9. Nemčovičová I, Benedict CA and Zajonc DM: Structure of human cytomegalovirus UL141 binding to TRAIL-R2 reveals novel, non-canonical death receptor interactions. PLoS Pathog 9: e1003224, 2013

10. Hsu JL, van den Boomen DJ, Tomasec P, Weekes MP, Antrobus R, Stanton RJ, Ruckova E, Sugrue D, Wilkie GS, Davison AJ, et al: Plasma membrane profiling defines an expanded class of cell surface proteins selectively targeted for degradation by HCMV US2 in cooperation with UL141. PLoS Pathog 11: e1004811, 2015.

11. Murrell I, Wilkie GS, Davison AJ, Statkute E, Fielding CA, Tomasec P, Wilkinson GW and Stanton RJ: Genetic stability of bacterial artificial chromosome-derived human cytomegalovirus during culture in vitro. J Virol 90: 3929-3943, 2016.

12. Wang G, Ren G, Cui X, Lu Z, Ma Y, Qi Y, Huang Y, Liu Z, Sun Z and Ruan Q: Human cytomegalovirus RL13 protein interacts with host NUDT14 protein affecting viral DNA replication. Mol Med Rep 13: 2167-2174, 2016.

13. Ross SA and Boppana SB: Congenital cytomegalovirus infection: Outcome and diagnosis. Semin Pediatr Infect Dis 16: 44-49, 2005.

14. Wang G, Ren G, Cui X, Lu Z, Ma Y, Qi Y, Huang Y, Liu Z, Sun Z and Ruan Q: Host protein Snapin interacts with human cytomegalovirus pUL130 and affects viral DNA replication. J Biosci 41: 173-182, 2016.

15. McMahon TP and Anders DG: Interactions between human cytomegalovirus helicase-primase proteins. Virus Res 86: 39-52, 2002.
16. Livak KJ and Schmittgen TD: Analysis of relative gene expression data using real-time quantitative PCR and the 2(-Delta Delta C(T)) method. Methods 25: 402-408, 2001.

17. Zavattoni M, Rustico M, Tassis B, Lombardi G, Furione M, Piralla A and Baldanti F: Risk of congenital disease in 46 infected fetuses according to gestational age of primary human cytomegalovirus infection in the mother. J Med Virol 88: 120-126, 2016

18. Xu LL, Mou WF, Yang L and Wang YC: Application of HCMV DNA detection in infants' blood, urine and mothers' breast milk in the diagnosis of HCMV infection among infants. Zhongguo Dang Dai Er Ke Za Zhi 15: 748-750, 2013 (In Chinese).

19. Choudhary A, Pati SK, Patro RK, Deorari AK and Dar L: Comparison of conventional, immunological and molecular techniques for the diagnosis of symptomatic congenital human cytomegalovirus infection in neonates and infants. Indian J Med Microbiol 33 (Suppl): 15-19, 2015.

20. Noriega V, Redmann V, Gardner T and Tortorella D: Diverse immune evasion strategies by human cytomegalovirus. Immunol Res 54: 140-151, 2012.

21. Wilkinson GW, Tomasec P, Stanton RJ, Armstrong M, Prod'homme V, Aicheler R, McSharry BP, Rickards CR, Cochrane D, Llewellyn-Lacey S, et al: Modulation of natural killer cells by human cytomegalovirus. J Clin Virol 41: 206-212, 2008.

22. Smith W, Tomasec P, Aicheler R, Loewendorf A, Nemčovičová I, Wang EC, Stanton RJ, Macauley M, Norris P, Willen L, et al: Human cytomegalovirus glycoprotein UL141 targets the TRAIL death receptors to thwart host innate antiviral defenses. Cell Host Microbe 13: 324-335, 2013.

23. David R: Immune evasion: UL141 keeps HCMV in charge. Nat Rev Microbiol 11: 297, 2013.

24. Loria PM, Duke A, Rand JB and Hobert O: Two neuronal, nuclear-localized RNA binding proteins involved in synaptic transmission. Curr Biol 13: 1317-1323, 2003.

25. Ladd AN, Charlet N and Cooper TA: The CELF family of RNA binding proteins is implicated in cell-specific and developmentally regulated alternative splicing. Mol Cell Biol 21: 1285-1296, 2001.

26. Dasgupta $\mathrm{T}$ and Ladd AN: The importance of CELF control: Molecular and biological roles of the CUG-BP, Elav-like family of RNA-binding proteins. Wiley Interdiscip Rev RNA 3: 104-121, 2012.

27. Gallo JM and Spickett C: The role of CELF proteins in neurological disorders. RNA Biol 7: 474-479, 2010.

28. Barron VA, Zhu H, Hinman MN, Ladd AN and Lou H: The neurofibromatosis type I pre-mRNA is a novel target of CELF protein-mediated splicing regulation. Nucleic Acids Res 38: 253-264, 2010

29. Kalsotra A, Xiao X, Ward AJ, Castle JC, Johnson JM, Burge CB and Cooper TA: A postnatal switch of CELF and MBNL proteins reprograms alternative splicing in the developing heart. Proc Natl Acad Sci USA 105: 20333-20338, 2008.

30. Vlasova-St Louis I, Dickson AM, Bohjanen PR and Wilusz CJ: CELFish ways to modulate mRNA decay. Biochim Biophys Acta 1829: 695-707, 2013.

31. Kline RA, Kaifer KA, Osman EY, Carella F, Tiberi A, Ross J, Pennetta G, Lorson CL and Murray LM: Comparison of independent screens on differentially vulnerable motor neurons reveals alpha-synuclein as a common modifier in motor neuron diseases. PLoS Genet 13: e1006680, 2017.

32. Anderson KN, Baban D, Oliver PL, Potter A and Davies KE: Expression profiling in spinal muscular atrophy reveals an RNA binding protein deficit. Neuromuscul Disord 14: 711-722, 2004.

33. Wu J, Li C, Zhao S and Mao B: Differential expression of the Brunol/CELF family genes during Xenopus laevis early development. Int J Dev Biol 54: 209-214, 2010. 